\title{
AVALIAÇÃO DE PARÂMETROS HIDRÁULICOS PARA MODELOS DE DISTRIBUIÇÃO DE ÁGUA NO SOLO SOB GOTEJAMENTO'
}

\author{
EUGÊNIO FERREIRA COÊLHO², DANI OR ${ }^{3}$ e VALDEMÍCIO FERREIRA DE SOUSA ${ }^{4}$
}

\begin{abstract}
RESUMO - As soluções analíticas de distribuição de água para ponto fonte e regime de fluxo nãopermanente são dependentes de parâmetros de solos considerados constantes em suas deduções. Erros na determinação desses parâmetros implicam insucesso dessas soluções. Este trabalho teve por objetivo avaliar o comportamento dos parâmetros $\alpha$ da equação de Gardner $\mathrm{k}(\mathrm{h})=\mathrm{K}_{\mathrm{s}} \mathrm{e}^{\alpha \mathrm{h}}$ e o parâmetro de linearização $\mathrm{k}=\mathrm{dK}(\theta) / \mathrm{d} \theta$ em diferentes posições do bulbo molhado, considerando os processos de infiltração isolado e seguido de redistribuição de água, à semelhança de um ciclo de irrigação. Dados de potencial matricial foram coletados em diversas posições do bulbo molhado em duas situações:(i) início da irrigação até atingir regime permanente em todo o bulbo molhado (infiltração); e (ii) durante dois ciclos de irrigação envolvendo infiltração e redistribuição de água. Os resultados mostraram que os parâmetros $\alpha$ e k variaram nas posições do bulbo molhado em relação ao gotejador, de acordo com o regime de umidade a que tais posições estiveram sujeitas. A obtenção desses parâmetros pelo método inverso requer testes que considerem as fases de infiltração e redistribuição em pelo menos dois ciclos de irrigação.
\end{abstract}

Termos para indexação: parâmetros físico-hídricos do solo, regime de fluxo não-permanente, relação solo-água, modelos analíticos.

\section{EVALUATION OF SOIL HYDRAULIC PARAMETERS FOR SOIL WATER DISTRIBUTION MODELS UNDER DRIP IRRIGATION}

\begin{abstract}
The transient analytical solutions for water distribution from point source are dependent on the soil parameters $\alpha$ from Gardner equation, i.e., $\mathrm{k}(\mathrm{h})=\mathrm{K}_{\mathrm{s}} \mathrm{e}^{\alpha \mathrm{h}}$ and the linearization parameter $\mathrm{k}=\mathrm{dK}(\theta) / \mathrm{d} \theta$ assumed constant in the derivation of these solutions. Errors in obtaining these parameters result in failure of the solutions. The objective of this work was to evaluate the parameter $\alpha$ and $\mathrm{k}$ at different locations in the wetted volume under an isolated and a cyclic infiltration process. Matric potential data were collected in several positions of the wetted volume in two situations: (i) beginning of irrigation until steady-state, i.e., only during infiltration process, and (ii) during two irrigation cycles involving infiltration and redistribution processes. The results showed that $\alpha$ and $\mathrm{k}$ parameters vary at different locations of the wetted volume related to the dripper according to the water regime at these locations. The use of inverse method for obtaining these parameters requires matric potential data from infiltration and redistribution during at least two irrigation cycles.
\end{abstract}

Index terms: soil physical parameters, soil-water relationship, analytical models, transient flow regime.

\footnotetext{
${ }^{1}$ Aceito para publicação em 7 de julho de 1998.

${ }^{2}$ Eng. Agríc., Ph.D., Embrapa-Centro Nacional de Pesquisa de Mandioca e Fruticultura (CNPMF), Rua Embrapa s/n, Caixa Postal 007, CEP 44380-000 Cruz das Almas, BA. E-mail: ecoelho@cnpmf.embrapa.br

${ }^{3}$ Eng. Agr., Ph.D., Utah State University. Logan, UT, 84322-4820, USA.

${ }^{4}$ Eng. Agr., M.Sc., Embrapa-Centro de Pesquisa Agropecuária do Meio Norte (CPAMN), Av. Duque de Caxias, 5650, CEP 64006-220 Teresina, PI.
}

\section{INTRODUÇÃO}

A irrigação localizada em todo o mundo tem aumentado de modo contínuo nos últimos anos, sendo esperado atingir ou exceder a três milhões de hectares no ano 2000 (Bucks, 1995). No Brasil, a expansão da irrigação localizada não só tem sido contínua, como tende a acelerar, em decorrência, principalmente, da expectativa de aumento das áreas plantadas com fruteiras em diversas regiões, em especial no Nordeste.

A irrigação por gotejamento, pelas suas características inerentes de alta uniformidade de aplicação 
de água e manutenção contínua de ótimos teores no solo, próximo ao sistema radicular, tem sido um dos métodos mais utilizados. Os altos investimentos requeridos na implantação desse sistema de irrigação localizada poderão, entretanto, não ser compensados se não forem utilizadas técnicas adequadas de manejo de irrigação que visem à racionalização do uso da água e aumento da produtividade.

O conhecimento da distribuição de água no volume molhado sob gotejamento é essencial na determinação do quanto irrigar e do momento da irrigação. $\mathrm{O}$ uso de modelos para descrever ou estimar a distribuição de água no volume molhado pode ser uma importante alternativa na definição do manejo da irrigação, permitindo até mesmo antecipar resultados de produção para diferentes opções de manejo.

Modelos numéricos a analíticos vêm sendo usados para descrever ou estimar a distribuição de água no bulbo molhado. Os modelos numéricos (Brandt et al., 1971; Lafolie et al., 1989; Claustnizer \& Hopmans, 1994) são razoavelmente precisos, mas requerem vários parâmetros de entrada e não permitem visualização direta do processo em questão. Os modelos analíticos (Raats, 1971; Lomen \& Warrick, 1974; Warrick, 1974; Bresler, 1978; Revol et al., 1995) são, em geral, mais limitados pelo número de variáveis que envolvem, entretanto, requerem menor número de parâmetros de entrada e são representados por equações mais acessíveis que as usadas nos modelos numéricos. Em geral, todos os modelos de distribuição de água no bulbo molhado consistem em soluções da equação da continuidade ou de Richards:

\section{$\frac{\partial \theta}{\partial \mathrm{t}}=\nabla(\mathrm{K}(\mathrm{h}) \nabla \mathrm{H})$}

onde: $\theta$ corresponde ao teor de água do solo; $\mathrm{h}$ ao potencial matricial; $\mathrm{H}$ ao potencial total, $\mathrm{t}$ ao tempo e $\mathrm{K}(\mathrm{h})$ à condutividade hidráulica não saturada. A solução desta equação pode ser obtida usando-se duas importantes ferramentas: (i) o modelo exponencial de Gardner (1958):

$\mathrm{K}(\mathrm{h})=\mathrm{K}_{\mathrm{s}} \mathrm{e}^{\alpha \mathrm{h}}$

em que $\alpha$ numa primeira instância é um parâmetro constante para determinado tipo de solo e $\mathrm{K}_{\mathrm{s}}$ é a condutividade hidráulica saturada; (ii) uma transformação definida por Philip (1971) da forma: $\theta=\int_{-\infty}^{\mathrm{h}} \mathrm{K}(\mathrm{h}) \mathrm{dh}=\frac{\mathrm{K}(\mathrm{h})}{\alpha}$

onde $\phi$ corresponde ao potencial matricial de fluxo $\left(\mathrm{L}^{2} \mathrm{~T}^{-1}\right)$. Warrick (1974) usou ainda um parâmetro $\mathrm{k}=\mathrm{dK} / \mathrm{d} \theta(\theta=$ teor de água do solo $)$, considerado constante para um determinado tipo de solo, obtendo a seguinte equação:

$$
\frac{\partial \phi}{\partial \mathrm{t}}=\frac{\mathrm{k}}{\partial} \nabla^{2} \phi-\mathrm{k} \frac{\partial \phi}{\partial \mathrm{t}}
$$

Warrick (1974) utilizou as variáveis adimensionais $\mathrm{R}=\alpha \mathrm{r} / 2, \mathrm{Z}=\alpha \mathrm{z} / 2, \mathrm{~T}=\alpha \mathrm{kt} / 4, \mathrm{r}=\left(\mathrm{R}^{2}+\mathrm{Z}^{2}\right)^{1 / 2}, \mathrm{e}$ $\Phi=8 \phi \pi / \alpha q,(q=$ vazão do emissor) para solucionar a equação 4 sujeita à condição inicial $\phi(r, z, 0)=0$ e à condição de contorno $(\partial \phi / \partial z)+\partial \phi=0$ em $z=0, r \neq 0$, obtendo a seguinte solução para ponto fonte abaixo da superfície do solo:

$\theta_{B}(R, Z, T)=\frac{e^{Z}}{2 \rho}\left\{e^{\rho} \operatorname{erf}\left(\frac{\rho}{2 \sqrt{T}}+\sqrt{T}\right)+e^{-\rho} \operatorname{erf}\left(\frac{\rho}{2 \sqrt{T}}-\sqrt{T}\right)\right\}$

e a solução, considerando ponto fonte à superficíe do solo:

$\theta(R, Z, T)=2\left\{\theta_{B}-e^{2 Z} \int_{Z}^{\infty} e^{-2 Z}\left[\theta_{B}\right]_{Z=Z} d Z\right\}$

em que a integral da equação (6) pode ser resolvida usando a fórmula de 15 pontos de Gauss-Leguerre (Sen et al., 1992).

A distribuição de $\phi(\mathrm{r}, \mathrm{z}, \mathrm{t})$ ao longo do tempo, simulando infiltração e redistribuição de água durante e após a irrigação, pode ser obtida por meio da superposição das soluções apresentadas, uma vez que essas são lineares, gerando a seguinte expressão:

$\theta(R, Z, T)=\frac{\alpha}{8 \pi} \sum_{i=0}^{n}\left(q_{i}-q_{i-1}\right) \theta\left(R, Z, T-T_{i}\right)$

com q $-1=0, \mathrm{~T}_{0}=0$ e $\mathrm{T}>\mathrm{T}_{\mathrm{i}}$.

Uma vez obtidas as soluções para distribuição de $\phi$ num plano bidimensional, o valor do potencial matricial numa certa posição $\mathrm{h}(\mathrm{r}, \mathrm{z}, \mathrm{t})$ do bulbo molhado pode ser determinado usando a transformação:

$\mathrm{h}(\mathrm{r}, \mathrm{z}, \mathrm{t})=\frac{1}{\alpha} \operatorname{In}\left[\frac{\alpha \theta}{\mathrm{K}_{\mathrm{s}}}\right]$

$\mathrm{O}$ modelo analítico apresentado tem sido avaliado (Ben-Asher et al., 1978; Sen et al., 1992; Angelakis 
et al., 1993) e recomendado para o dimensionamento de sistemas de irrigação por gotejamento (Amoozegar-Fard et al., 1984; Warrick, 1986), entretanto esse modelo não tem ainda atingido larga aplicação prática.

Tendo em vista que as variáveis envolvidas no modelo se constituem basicamente nos parâmetros hidráulicos do solo $\alpha, \mathrm{K}_{\mathrm{s}}$ e k=dK/d $\theta$, a precisão na determinação dessas variáveis é crucial no sucesso do uso do modelo.

O parâmetro $\alpha$ é uma constante empírica relacionada à porosidade do solo ou às suas propriedades de retenção de água, cujos valores variam de $0,002 \mathrm{~cm}^{-1} \mathrm{em}$ solo de textura fina a $0,8 \mathrm{~cm}^{-1} \mathrm{em}$ solo de textura arenosa (Philip, 1969). O parâmetro k pode ser determinado a partir de curvas de $\mathrm{k}(\theta) \times \theta$, pela equação $\mathrm{k}=\alpha \mathrm{D}(\theta)$, sendo $\mathrm{D}(\theta)$ a difusividade $\left(\mathrm{L}^{2} \mathrm{~T}^{-1}\right)$ representante de uma faixa de valores de $\theta$, ou de modo empírico, mediante ajuste dos valores de h ou $\theta$ calculados e dos valores medidos (Ben-Asher et al., 1978). Os parâmetros $\alpha$ e $\mathrm{K}_{\mathrm{s}}$ podem ser determinados por diferentes métodos de campo ou de laboratório (Stephens, 1985; Shani et al., 1987; White \& Perroux, 1987). Muitos desses métodos têm por base a solução aproximada de Wooding (1968) para infiltração a partir da superfície do solo irrigada por emissão de água originada de um ponto fonte. Esses parâmetros podem ser, também, determinados pelo método inverso, no qual as variáveis fins (h e $\theta$ ) são conhecidas e as variáveis meio $\left(\alpha\right.$ e $\left.\mathrm{K}_{\mathrm{s}}\right)$ são desconhecidas e determinadas por um processo de otimização (Russo et al., 1991).

Os parâmetros $\alpha$ e k são considerados constantes para um certo tipo de solo, entretanto, Parlange $\&$ Hogarth (1985) observaram que o valor de $\alpha$, na média, pode ser superior em todos os solos de textura arenosa, e que em solos com teores de água variando de seco a saturado esta afirmativa não é verdadeira. Tal comportamento é conseqüência do fato de a equação de Gardner (1958) ser tipicamente uma boa aproximação da condutividade hidráulica nãosaturada para intervalos de $\mathrm{h}$, representando altos teores de água, não representando, portanto, a função $\mathrm{K}(\mathrm{h}) \times \mathrm{h}$ em todas as possíveis faixas desses valores (Moldrup et al., 1989). O valor de k, determinado para certa faixa de valores de h ou $\theta$, numa dada posição do bulbo molhado não deve ser extrapolado para outras posições do bulbo (Ben-Asher et al., 1978), pois possíveis variações podem ocorrer com o processo de infiltração e redistribuição no bulbo molhado. A flexibilidade desses parâmetros de solo pode influir consideravelmente no uso prático do modelo de Warrick (1974), necessitando portanto atenção especial.

O presente trabalho teve por objetivo avaliar o comportamento dos parâmetros $\alpha$ e k em diferentes posições do bulbo molhado, considerando o processo de infiltração isolado e seqüenciado pela redistribuição de água à semelhança de um ciclo de irrigação.

\section{MATERIAL E MÉTODOS}

Dois experimentos foram conduzidos em casa de vegetação, utilizando-se um solo franco siltoso $\left(290 \mathrm{~g} \mathrm{~kg}^{-1} \mathrm{de}\right.$ areia, $550 \mathrm{~g} \mathrm{~kg}^{-1}$ de silte, $160 \mathrm{~g} \mathrm{~kg}^{-1}$ de argila, porosidade total média de $0,46 \mathrm{~cm}^{3} \mathrm{~cm}^{-3}$, densidade global de $1,37 \mathrm{~kg} \mathrm{dm}^{-3}$ ). Os valores médios de $\mathrm{K}_{\mathrm{s}}$ foram $0,049 \mathrm{~m} \mathrm{~h}^{-1}$ e $0,025 \mathrm{~m} \mathrm{~h}^{-1}$ determinados à superfície do solo e a $0,3 \mathrm{~m}$ de profundidade, respectivamente.

A Fig. 1 apresenta as características de retenção de água do solo, em que se usou o modelo de Russo (1988) para ajuste da curva aos dados. A irrigação foi por gotejamento, com gotejadores autolimpantes e autocompensantes de vazão $1,6 \mathrm{~L} \mathrm{~h}^{-1}$ instalados no centro de duas caixas de paredes de acrílico, de $0,8 \mathrm{~m}$ x $0,8 \mathrm{~m} \times 1,0 \mathrm{~m}$. Numa caixa, o gotejador foi localizado à superfície do solo

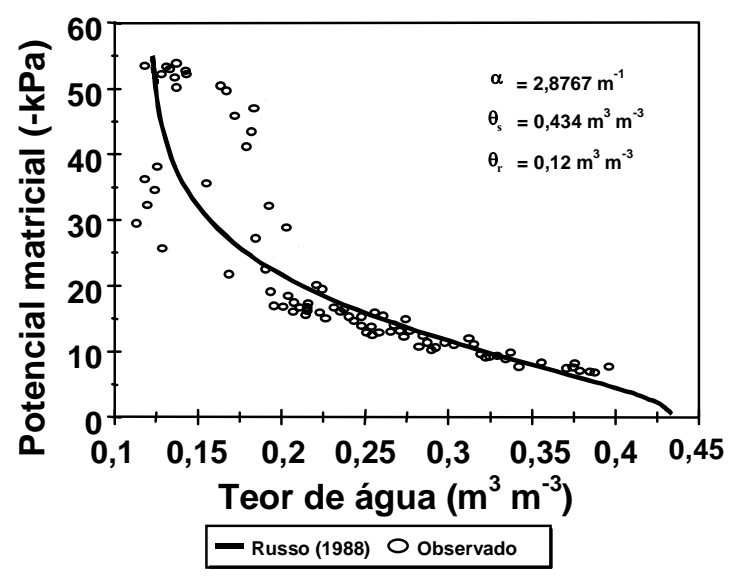

FIG. 1. Curva de retenção de água do solo ajustada aos dados de teor de água e potencial matricial conforme Russo (1988). 
e na outra a $0,3 \mathrm{~m}$ abaixo da superfície do solo. Os dados de potencial matricial foram coletados em 16 pontos num perfil radial ao gotejador às distâncias de $0,1 \mathrm{~m} ; 0,2 \mathrm{~m}$; $0,3 \mathrm{~m}$ e $0,4 \mathrm{~m}$ na horizontal (r), e $0,1 \mathrm{~m} ; 0,3 \mathrm{~m} ; 0,5 \mathrm{~m}$ e $0,7 \mathrm{~m}$ na vertical em relação à superfície do solo $(\mathrm{z})$.

O monitoramento dos potenciais matriciais foram feitos por meio de tensiômetros ligados por transdutores de pressão a um multiplicador de leituras acoplado a um "datalogger". No primeiro experimento, manteve-se o gotejador (à superfície do solo ou enterrado) em funcionamento até se observar condição de regime de fluxo permanente em todas as posições do perfil. Em seguida, após o solo das caixas voltar à condição inicial $(-88,2 \mathrm{kPa})$ foi executado o segundo experimento, promovendo-se irrigações com tempo de aplicação de seis horas e intervalos de dois dias. Em ambos experimentos, a evaporação foi evitada cobrindo-se a superfície do solo com material do tipo esponja envolvido com papel alumínio.

A determinação dos parâmetros $\alpha$ e $\mathrm{k}$ foi feita pelo método inverso usando-se um processo de otimização executado com base na minimização da soma de quadrados dos valores de potencial matricial medidos desde o início da aplicação de água até se atingir regime de fluxo permanente e estimados pelas equações 5,6 e 8 para gotejador à superfície do solo e enterrado. No processo de otimização o valor de $\mathrm{K}_{\mathrm{s}}$ foi assumido constante, ficando como variáveis do processo $\alpha$ e $\mathrm{k}$.

\section{RESULTADOS E DISCUSSÃO}

\section{Avaliação de $\alpha$ e k considerando somente infiltra- ção}

A Fig. 2 ilustra um exemplo dos resultados dos ajustes dos modelos aos dados de potencial matricial para algumas posições do perfil. Observa-se que, tanto no caso do gotejador à superfície do solo como enterrado, houve uma tendência de redução dos valores de $\alpha$ e k em relação a maiores distâncias do gotejador. Esses resultados mostram que os parâmetros em estudo, mesmo num solo considerado homogêneo, não devem ser tomados como constantes, o que reforça as conclusões de Parlange \& Hogarth (1985) sobre o parâmetro $\alpha$, e as de BenAsher et al. (1978) sobre o parâmetro k.

Os valores de $\alpha$ não podem ser baseados exclusivamente em características texturais do solo. Há indícios de o parâmetro estar relacionado à porosidade livre de água, ou seja, quanto maior a porosidade de um solo, menor o valor desse parâmetro. Isto pode, também, ser observado na curva K(h) x h (Fig. 3), em que $\alpha$ é a declividade e se reduz à medida que $h$ decresce, ou seja, para faixas de baixos potenciais matriciais, onde a porosidade livre de água é maior, os valores de $\alpha$ são menores.

As variações dos valores de k são esperadas, uma vez que o tempo de chegada da água e a taxa de umedecimento numa dada posição do perfil depende das coordenadas $r$ e z em relação ao gotejador.
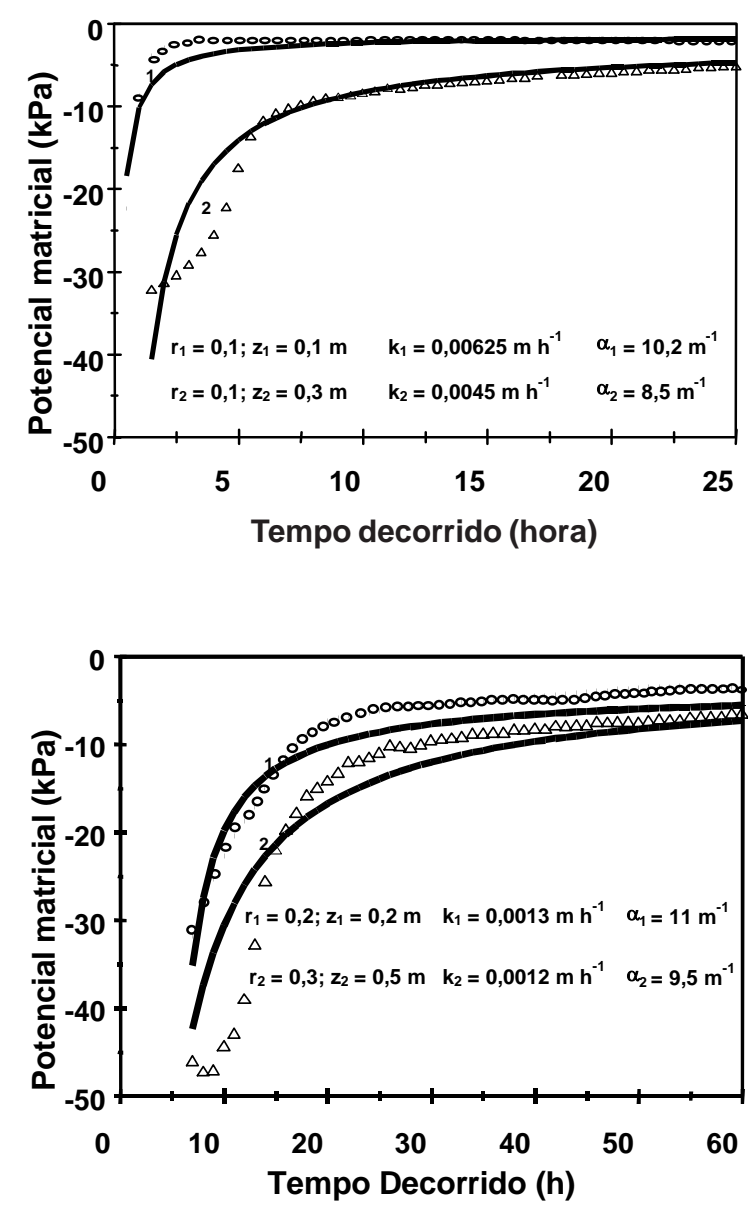

FIG. 2. Ajuste dos dados de potencial matricial aos valores calculados pela solução analítica de Warrick (1974) tomados durante o processo de infiltração até se atingir regime de fluxo permanente considerando o gotejador (a) à superfície do solo e (b) a $0,3 \mathrm{~m}$ de profundidade. 


\section{Caso de infiltrações cíclicas (irrigação)}

Os ajustes do modelo de Warrick (1974), que envolve todas as equações $5,6,7$ e 8 , aos dados de potencial matricial ao longo de dois ciclos de irrigação em relação a algumas posições do bulbo molhado são mostrados na Fig. 4 (gotejador à superfície do solo) e Fig. 5 (gotejador a $0,3 \mathrm{~m}$ de profundidade). Os valores dos parâmetros $\alpha$ e k obtidos evidenciaram dois importantes aspectos: (i) a diferença entre os valores de $\alpha$ e k obtidos considerando o processo de infiltração isolado e cíclico numa mesma posição do bulbo molhado; (ii) a variação de $\alpha$ e k nas diferentes posições em relação ao gotejador. No primeiro caso, a inclusão do processo de redistribuição, que funciona de modo inverso à infiltração, é o principal fator que promove a diferença entre os parâmetros de solo. Nota-se que os valores de $\alpha$ e de $\mathrm{k}$, no caso da irrigação (infiltrações cíclicas), foram menores e maiores, respectivamente, quando comparados aos mesmos parâmetros no caso da infiltração isolada. A variação entre os parâmetros nas diferentes posições do perfil, tanto com o gotejador à superfície do solo como enterrado, manteve a mesma tendência observada no processo de infiltração isolado, ou seja, os maiores valores dos parâmetros corresponderam às posições mais próximas do gotejador.

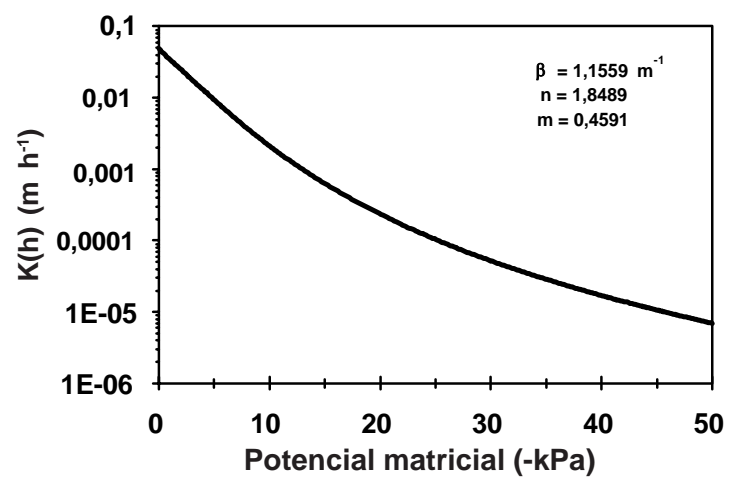

FIG. 3. Curva da condutividade hidráulica nãosaturada em função do potencial matricial do solo obtida pelo modelo de Van Genuchten (1980).

\section{Obtenção e uso de $\alpha$ e k no modelo de Warrick (1974)}

Os resultados evidenciaram que se deve ter precauções no uso das soluções analíticas de distribuição de água no solo linearizadas por meio da equação de Gardner (1958), que não é válida considerando os mesmos parâmetros hidráulicos em todas as faixas de potenciais matriciais do solo. No caso do modelo de Warrick (1974), acrescenta-se a limitação inerente e indispensável do parâmetro de linearização $\mathrm{k}(\mathrm{dK} / \mathrm{d} \theta)$ considerado constante. Num perfil de solo inserido no bulbo molhado, os valores de $\alpha$ e $\mathrm{k}$ nas regiões mais próximas do emissor serão provavelmente diferenciados dos valores nas regiões mais afastadas. Deve-se ater, também, na determinação desses parâmetros pelo método inverso, com fins práticos de irrigação, por exemplo, à realização de
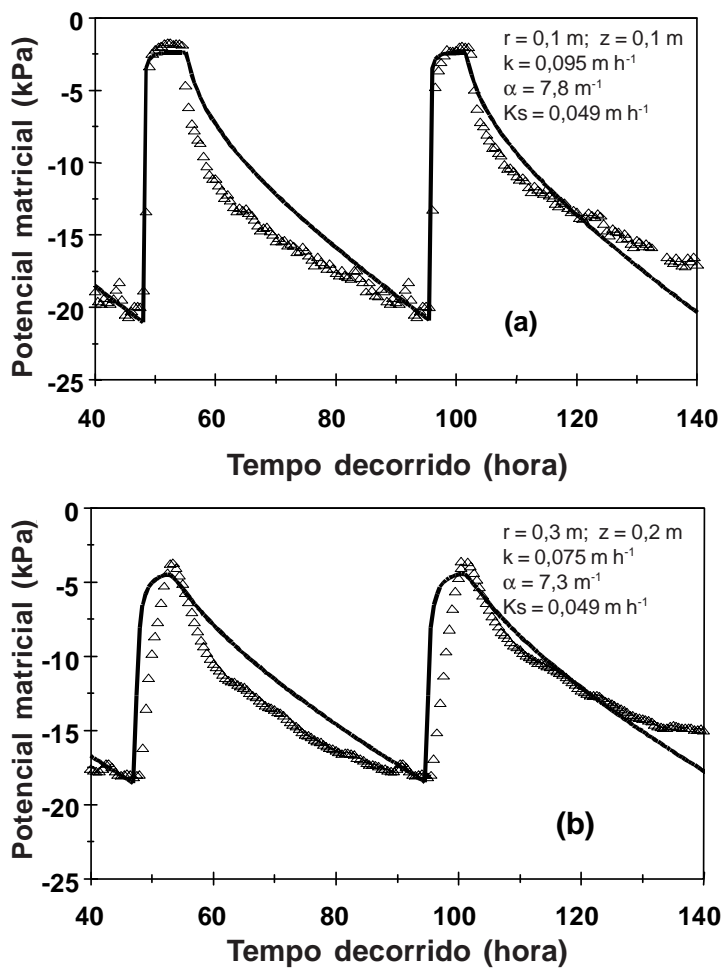

FIG. 4. Ajuste otimizado dos valores do potencial matricial medidos e calculados pelo modelo de Warrick (1974) ao longo de dois ciclos de irrigação, com o gotejador à superfície do solo.

Pesq. agropec. bras., Brasília, v.34, n.4, p.651-657, abr. 1999 

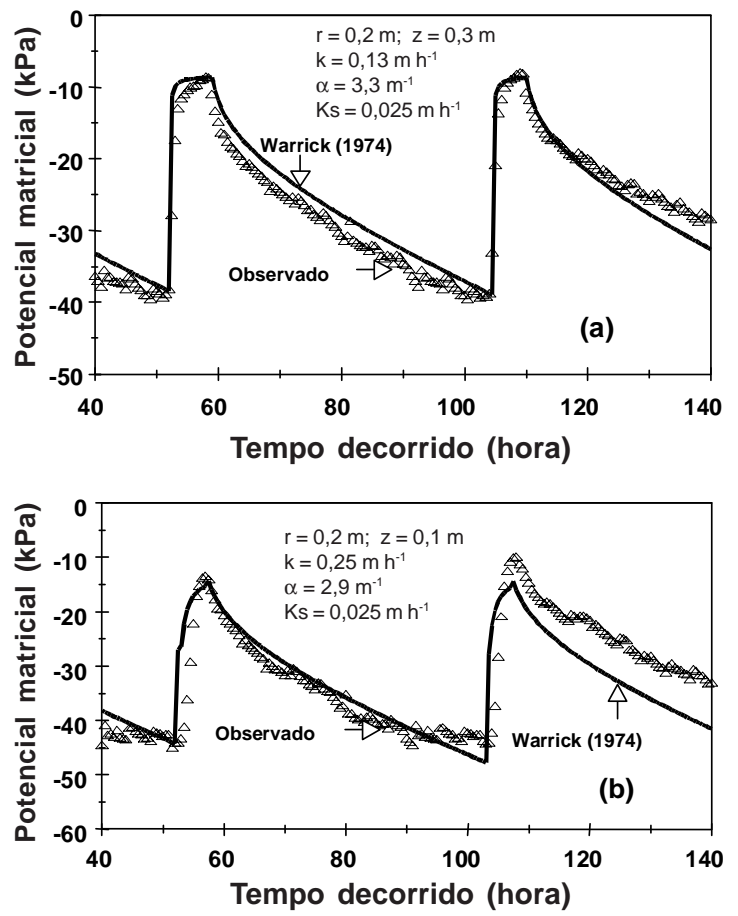

FIG. 5. Ajuste otimizado dos valores do potencial matricial medidos e calculados pelo modelo de Warrick (1974) ao longo de dois ciclos de irrigação, com o gotejador enterrado a $0,3 \mathrm{~m}$.

testes considerando infiltrações cíclicas, ou seja, as fases de infiltração e redistribuição em pelo menos dois ciclos de irrigação. Uma alternativa para a determinação de $\alpha$ quando se dispõe da curva $\mathrm{K}(\mathrm{h}) \mathrm{x}$ h para o solo em questão é o uso do método de Moldrup et al. (1989), que considera a variação de $\alpha$ para diferentes faixas de potenciais matriciais, conforme usado por Coelho \& Or (1996).

\section{CONCLUSÕES}

1. Os parâmetros $\alpha$ e k variam nas diferentes posições no bulbo molhado em relação ao gotejador, de acordo com o regime de umidade a que as posições estão sujeitas.

2. Os maiores valores dos parâmetros $\alpha$ e k no bulbo molhado, tanto num processo de infiltração isolado como cíclico, correspondem às posições mais próximas do gotejador.
3. Os valores de $\alpha$ não devem ser fundamentados, exclusivamente, em características texturais do solo.

4. O método inverso para determinação de $\alpha$ e k para fins de irrigação requer coleta de dados em testes que envolvam as fases de infiltração e redistribuição em pelo menos dois ciclos de irrigação.

\section{REFERÊNCIAS}

AMOOZEGAR-FARD, A.; WARRICK, A. W.; LOMEN, D.O. Design nomographs for trickle and subsurface irrigation. Journal of Irrigation and Drainage Division of the American Society of Civil Engineering, v.110, p.107-120, 1984.

ANGELAKIS, A.N.; ROLSTON, D.E.; KADIR, T.N.; SCOTT, V.H. Soil water distribution under trickle source. Journal of Irrigation and Drainage Division of the American Society of Civil Engineering, v.119, p.484-497, 1993.

BEN-ASHER, J.; LOMEN, D.O.; WARRICK, A.W. Linear and nonlinear models of infiltration from a point source. Soil Science Society of America. Journal, Madison, v.42, p.3-6, 1978.

BRANDT, A.; BRESLER, E.; DINER, N.; BEN-ASHER, J.; HELLER, J.; GOLDBERG, D. Infiltration from a trickle source: I. Mathematical models. Soil Science Society of America. Proceedings, Madison, v.35, p.675-694, 1971.

BRESLER, E. Analysis of trickle irrigation with application to design problems. Irrigation Science, New York, v.1, p.3-17, 1978.

BUCKS, D.A. Historical development in microirrigation. In: INTERNATIONAL MICROIRRIGATION CONGRESS, 5., 1995, Orlando. Proceedings..., St. Joseph: American Society of Agricultural Engineering, 1995. p.1-5.

CLAUSTNIZER, V.; HOPMANS, J.W. Simultaneous modeling of transient three-dimensional root growth and soil water flow. Plant and Soil, The Hague, v.164, p.299-314, 1994.

COELHO, E.F.; OR, D. Soil water dynamics under drip irrigation: transient flow and uptake models. Transactions of the ASAE, St. Joseph, v.39, p.20172025, 1996.

GARDNER, W.R. Some steady state solutions of the unsaturated moisture flow equation with application 
to evaporation from water table. Soil Science, Baltimore, v.85, p.228-232, 1958.

LAFOLIE, F.; GUENNELON, R.; VAN GENUCHTEN, M.T. Analysis of water flow under trickle irrigation: II. Experimental evaluation. Soil Science Society of America. Journal, Madison, v.53, p.1318-1323, 1989.

LOMEN, D.O.; WARRICK, A.W. Time-dependent linearized infiltration: II. Line sources. Soil Science Society of America. Proceedings, Madison, v.38, p.568-572, 1974.

MOLDRUP, P.; ROLSTON, D.E.; HANSEN, J.A. Rapid and numerically stable simulation of onedimensional, transient water flow in unsaturated layered soils. Soil Science, Baltimore, v.148, p.219226, 1989.

PARLANGE, J.Y.; HOGARTH, W.L. Steady-state infiltration: consequences of dependent on soil moisture content. Water Resourouces Research, Washington DC, v.21, p.1283-1284, 1985.

PHILIP, J.R. Theory of infiltration. Advances Hydroscience, New York, v.5, p.215-296, 1969

PHILIP, J.R. General theorem on steady state infiltration from surface sources, with application to point and line sources. Soil Science Society of America. Proceedings, Madison, v.35, p.867-871, 1971.

RAATS, P.A.C. Steady infiltration from point sources, cavities and basins. Soil Science Society of America. Proceedings, Madison, v.35, p.689-694, 1971.

REVOL, P.; CLOTHIER, B.E.; LESAFFRE, B.; VACHAUD, G. An approximate time-dependent solution for point-source infiltration. In: INTERNATIONAL MICROIRRIGATION CONGRESS, 5., 1995, Orlando. Proceedings... St. Joseph: American Society of Agricultural Engineering, 1995. p.603-608.

RUSSO, D. Determining soil hydraulic properties by parameter estimation: on the selection of a model for hydraulic properties. Water Resourouces Research, Washington DC, v.24, p.453-459, 1988.
RUSSO, D.; BRESLER, E.; SHANI, U.; PARKER, J.C. Analysis of infiltration events in relation to determining soil hydraulic properties by inverse problem metodology. Water Resouruces Research, Washington DC, v.27, p.1361-1373, 1991.

SEN, H.S.; PAUL, D.; BANDYOPADHYAY, K.; DASH, N.B. A simple numerical solution for twodimensional moisture distribution under trickle irrigation. Soil Science, Baltimore, v.154, p.350356, 1992.

SHANI, U.; HANKS, R.J.; BRESLER, E.; OLIVEIRA, C.A.S. Field method for estimating hydraulic conductivity and matric potential-water content relations. Soil Science, Baltimore, v.51, p.298-302, 1987.

STEPHENS, D.B. A field method to determine unsaturated hydraulic conductivity using flow nets. Water Resources Research, Washington DC, v.21, p.45-50, 1985.

VAN GENUCHTEN, M.T. A closed-form equation for predicting the hydraulic conductivity of unsaturated. Soil Science Society of America. Journal, Madison, v.44, p.892-898, 1980.

WARRICK, A.W. Time-dependent linearized infiltration. I. Point sources. Soil Science Society of America. Proceedings, Madison, v.38, p.383-386, 1974.

WARRICK, A. W. Design principles. In: NAKAYAMA, F.S.; BUCKS, D.A. (Eds.). Trickle irrigation for crop productions. New York: Elsevier, 1986. p.93116.

WHITE, I.; PERROUX, K.M. Use of sorptivity to determine field soil hydraulic properties. Soil Science Society of America. Journal, Madison, v.51, p.1093-1101, 1987.

WOODING, R.A. Steady infiltration from a shallow circular pond. Water Resources Research, Washington DC, v.4, p.1259-1273, 1968. 